\title{
OLMRP: Hierarchical Outflow Load-balancing Multicast Routing Protocol for Congestion Control in Ad hoc Networks
}

\author{
G.S.Sreedhar \\ Department of CSE \\ K.O.R.M.College of Engineering , Kadapa
}

\author{
A.Damodaram, PhD. \\ Professor of CSE and Director, Academic \\ Audit Cell, JNTUH, Hyderabad
}

\begin{abstract}
This paper, proposes a new multicast routing protocol for mobile ad hoc networks that handles the congestion to improve the Quality of routing service. Part of the proposed protocol introduced a MAC layer level solution called Group Level Multicasting (GLM) that effectively handles the multicasting at one hop level mesh or tree. The proposed model controls the congestion in hierarchical order to minimize the resource utilization. Limited bandwidth and a high degree of mobility require that routing protocols for ad hoc networks be adaptive, trouble-free, and energy saving. This paper proposed a new Hierarchical Outflow Loadbalancing Multicast Routing Protocol (OLMRP) for Ad hoc networks, which handles congestion state. OLMR is capable to adapt a mesh or tree structure with enhanced similar resilience against mobility. OLMRP utilizes GLM to reduce the overhead of route failure recovery, improve route efficiency and reduce data transmissions. The simulation results show that OLMR handles congestion with reduced control overhead in various environments, also the improved packet delivery ratio.
\end{abstract}

Keywords: multicast, on-demand routing, congestion control, ad hoc network.ODMRP, OLMRP, MGCA, MGOL

\section{INTRODUCTION}

As described in [2], [3], the first objective of a multicast protocol is to convey packets from a source to the members of a multicast group with an acceptable quality of service (QoS). QoS offered by the network comes under performance, in general [1]. Specifically, QoS in voice communications necessitates 1) maintaining a high enough packet delivery ratio (PDR), 2) keeping the packet delay low enough, and 3) minimizing the jitter in packet arrival times. Thus, the goal in QoS provisioning is to Achieve a more deterministic network behavior termed as bounded delay, jitter, and PDR is a key factor for QoSprovisioning [1]. The simple group communication algorithm called flooding is good enough to attain high PDR provided that the data traffic and/or node density is not very high so that the network is not congested. Though, flooding usually is not ideal as a multicast routing protocol due to its excessive use of the available bandwidth. Thus, the second objective of a multicast routing protocol is to utilize the bandwidth efficiently, which is directly related with the number of retransmissions required to deliver generated data packets to all members of a multicast group with a maximum adequate PDR. The later intent of a multicasttopology is to minimize the energy dissipation of the network. Although optimizing the performance of a wireless communication system by incorporating cross-layer design is a tempting choice, several researchers have argued that such a cross-layer design is not the best choice in the long run because it sacrifices modularity and can lead to unintended cross-layer interactions [6]. However, by strictly adhering to a standard hierarchical scenario, it could be possible to miss out on performanceenhancements that can be offered through the exploitation of the less restricted cross-layer design space. Therefore, in this paper, a multicasting architecture is proposed that handles the congestion successfully. Although there are many protocols for multicasting in mobile ad hoc networks [4], [5], [7], [8], [9], to the best of my knowledge, there is no single protocol that is cross model for tree and mesh based architecture to handle congestion in order to improve the QOS. Thus, this paper, proposed such a distributed architecture multicast ad-hoc routing that handles the congestion with hierarchical outflow load balancing.

\section{RELATED WORK}

Congestion awareness and control in networks is the issue that attains reasonable attention from the domain of research.Xiaoqin Chen et al[2] described a congestion aware routing, which handles congestion by some selective metrics that are used to assess data-rate, MAC overhead and buffer delay, which helps to identify and deal the congestion contention area in the network. HongqiangZhai, et al., [3] proposed a solution by arguing that congestion and severe medium contention is interrelated.Yung Yi et al., [4] proposed a hop level congestion control approach. Tom Goff, Nael et al., [5] explored a set of algorithms that initiates alternative path usage when the quality of a path in use becomes suspect. Xuyang et al., [6] present a cross-layer hop-by-hop congestion control scheme designed to improve TCP performance in multi hop wireless networks. The impact of congestion on transport layer that degrades the performance was described in [7]. Duc et al., [8] argued that current designs for routing are not congestion adaptive.

Based on the studies in [6] [5], a loss-event based disconnection timer of ADMR is claimed to be problematic when used as an indicator of mobility since it triggers a source node to initiate a time limited data flood into the currently congested networks. The authors also, particularly, mentioned problems of Receiver join storms and ACK implosions of ADMR under high-density scenarios based on a static exhibition model. Solutions to both problems are proposed by setting a predefined minimum limit for Join, Repair, and Explicit ACK timers to allow data packets to be transmitted [5]. However, such fixed timeout value is sensitive to the protocol performance since using a large value for the RepairWait-Time can degrade ADMR efficiency under high mobility networks while a large value of Ack-Wait-Time can result in low throughput and excessive pruning. Moreover, the solutions are scenario specific as they are designed for singlegroup based multicast, in which all receivers are at one-hop away from the source and they must send explicit ACKs to 
maintain the forwarding status. Although network congestion is the dominant reason for packet loss in such scenarios, little effort has been seen trying to address the problem from a routing perspective [8][9][10]. In [8], the notion of exploiting congestion information in networks and higher layer protocols is used for unicast routing by focusing on an enhancement of the dynamic source routing (DSR) protocol. The evaluation demonstrates substantial performance improvement in terms of scalability, routing overhead and packet delivery ratio. A congestion-adaptive routing protocol (CRP) [10] is another unicast routing protocol using congestion information to help increase its effectiveness and efficiency. Based on a bypass routing concept, $\mathrm{CRP}$ is able to provide better performance in terms of delay variation in heavily loaded networks as compared to other outstanding on demand unicast routing protocols.

Most of the existing models are aimed to identify the congestion through packet loss. Most frequent times this packet loss can be an impact of disconnection in routing path. Hence an attempt to outflow load balancing to control the packet loss that occurs against link failure is a useless effort. The other expensive approach that opted by most of the existing solutions is regularizing the egress at all nodes taking part in routing. In general it is possible to control the congestion at hop level [4][15]. Hence egress regularization at each node of the network would be an expensive in resource utilization. This paper highlights an argument that it is an essential requirement to identify the reason for packet loss. Hence the solution can put efforts to reestablish the path against link failure conditions. And also this paper put forward the argument that hop level congestion control is not sufficient, thisis due to the inability of hop level nodes to balance the outflow load to control the congestion, the resource usage leftovers same as in source level egress regularization models.

A Multicast ad hoc routing topology is proposed here that controls the congestion through stratified egress tuning approach. The initial goal of the proposed model is congestion avoidance; hence a packet transmission strategy at MAC layer has been introduced that referred as Group Level Multicast (GLM) packet transmission.

\section{OLMRP}

The packet dropping often occurs in Manets. The reasons for this packet dropping are as below

$>$ Transmission Link failure.

$>$ Inferred Transmission due to overwhelming Inflow that leads Inflow load to low. This also can claim as packet dropping due to congestion at routing.

A hierarchical order is used to handle the congestion state as follows

$>$ The Status of congestion within Multicast Group

$>$ The status of congestion between Multicast Groups

This helps in minimizing of source level outflow regulation cost and balances the power consumption.

\subsection{Network and Node activities under proposed protocol:}

The network is to be split into Multicast groups with respect to nodes participating in multicast such that multicast nodes as multicast group heads
For each multicast group $i$ where $i=1 . .|M G|$; $(|M G|$ is the total number of multicast groups )

Find transmission load threshold $\zeta_{n}$ for each multicast group $i$

By using $\zeta_{n}$ of each multicast group Transmission load threshold for entire network can be measured .

\subsection{Information sharing within Multicast Group [ between Node and multicast group head]}

Each node $n$ that belongs to multicast group $M G_{i}$ verifies the outflow load and shares degree of outflow load $d(\mathrm{ol})_{n}$ with multicast group head. Once $n d(o l)_{k}$ received from each node $k$ of the multicast group $M G_{i}$, the multicast group head $M G_{i}(h)$ calculates the degree of outflow load mgd (Ol) ${ }_{\mathrm{MG}_{i}}$ at Multicast Group $M G_{i}$.

$\operatorname{mgd}(o l)_{M G_{i}}=\frac{\sum_{k=1}^{\left|M G_{i}\right|} n d(o l)_{k}}{\left|M G_{i}\right|}$

\subsection{Multicast Group Congestion Assessment(MGCA) Algorithm}

Multicast Group congestion assessment (MGCA) algorithm is presented in this section. MGCA helps in locating the stateof packet dropping due to congestion. This evaluation occurs under Mac layer. The algorithm MGCA follows

\section{MGCA Algorithm}

At an event of inflow loadat node $i$ :

Updating Inflow load:

$$
\begin{aligned}
& \text { if }\left(\left(\gamma_{t}-\gamma_{T}\right)<0\right) d o \\
& \sigma^{\prime}:=0.5 \times\left(\frac{i l_{c r}-i l_{\gamma_{T}}}{\gamma_{t}}\right) \\
& i l_{\gamma_{T}}:=i l_{c r}\left(\frac{\gamma_{t}}{\gamma_{T}}\right)+i l_{\gamma} \\
& \text { endif } \\
& \text { if }\left(\left(\gamma_{t}-\gamma_{T}\right) \geq 0\right) d o \\
& \sigma^{\prime}:=\frac{i l_{c r}-i l_{\gamma_{T}}}{\gamma_{t}} \\
& \text { il }{ }_{\gamma_{T}}:=i l_{c r} \\
& \text { endif }
\end{aligned}
$$$$
\sigma^{\prime}:=0.5 \times\left(\frac{i l_{c r}-i l_{\gamma_{T}}}{\gamma_{t}}\right)+0.5 \times\left(\sigma^{\prime}\right)
$$$$
i l_{\gamma_{T}}:=i l_{c r}\left(\frac{\gamma_{t}}{\gamma_{T}}\right)+i l_{\gamma_{T}}\left(\frac{\gamma_{T}-\gamma_{t}}{\gamma_{T}}\right)
$$

Here in the above conditional statement

$\gamma_{t}$ :Time between last two transmissions of hop level connected nodes in routing path 
$\gamma_{T}$ : Time between two transmissions of hop level connected nodes in routing path

$\sigma^{\prime}$ : Average slop threshold of the inflow load

$i l_{c r}:$ Current inflow load ratio

${ }^{i \gamma_{T}}$ : Average inflow load threshold observed for predefined interval $\gamma_{T}$

$i l_{c r}:$ Current inflow load ratio

${ }^{i l}{ }_{r}:$ Inflow load ratio

${ }^{i} l_{c e}$ : Expected inflow load threshold at current interval

Detecting packet drop at the Mac layer level:

$i l_{c e}=i l_{\gamma_{T}}+\sigma^{\prime} \gamma_{e t}$

if $\left(i l_{c e}<i l_{r}\right) d o$

packet loss due to link failure

else

packet loss due to congestion

endif

\subsection{Multicast Group Outflow Load-balancing(MGOL) Algorithm}

MGOL initiates if congestion found at a node $i$ in routing path. Upon receiving congestion alerts from Mac layer the routing protocol initiates MGOL. Let $S$ be the node that transmits data to hop level node $i$. If node $i$ affected by congestion, then MGOL alerts node $S$. Upon receiving alerts about the congestion state at the hop level target node $i, s$ evaluates ' $n d(o l)_{S}>m g d(o l)_{M G_{C}}$ ', if true then verifies that $\left(n d(o l){ }_{S}-m g d(o l){ }_{M G_{C}}\right)$ is greater than or equal to $\varepsilon_{s}$ is true or not. If true then the node $S$ balances its outflow load such that $n d(o l)_{s}$ is not less than $m g d(o l){ }_{M G}$

Here in the above description $\varepsilon_{s}$ is outflow threshold at node $s, M G_{c}$ is current multicast group such that $s \in M G_{c}$

The node $S$ balances its outflow load by increasing packet such that $n d(o l)_{s}$ is greater or equal to $\operatorname{mgd}(o l){ }_{M G_{C}}+\varepsilon_{M G_{C}}$

$\varepsilon_{M G_{C}}=\frac{\sum_{k=1}^{M G_{C} \mid} m g d(o l)_{M G_{C}}-n d(o l)_{k}\left\{k \in M G_{C} \text { and } \mathrm{k} \text { is a node }\right\}}{\left|M G_{c}\right|}$
If

$\left(n d(o l)_{s} \leq \operatorname{mgd}(o l)_{M G_{c}}\right) o r\left(\left(n d(o l)_{S}-\operatorname{mgd}(o l)_{M G_{C}}\right)<\varepsilon_{s}\right)$

' then node $s$ avoids balancing its outflow load and alerts the $M G_{c}(h)$ (multicast group head of the $M G_{c}, s \in M G_{c}$ ). Then $M G_{c}(h)$ alerts all upstream unicast nodes to the node $S$ of the multicast group $M G_{c}$. Upon receiving alerts from $M G_{c}(h)$ all upstream unicasting nodes attempts to balance their outflow load of the node $S$ and updates their ' $n d(\mathrm{ol})$ '. Each unicasting node that updated its ' $n d(o l)$ ' and alerts the $M G_{c}(h)$, then $M G_{c}(h)$ estimates $\operatorname{mgd}(o l)_{M G_{C}}$ and checks the same with $d(o l)$ as follows

$\operatorname{mgd}(o l)_{M G_{c}} \geq d(o l)+\bar{\varepsilon}$ is true or not.

Here in this equation $d(o l)$ is the routing path level degree of outflow load and $\bar{\varepsilon}$ is outflow load threshold measured at the path level.

If the given condition is true, then MGOL process ends, if not $M G_{c}(h)$ alerts $M G_{p}(h)$ then MGOL initiates at multicast group $M G_{p}$, which is adjacent upstream multicast group to $M G_{c}$. The MGOL process at $M G_{p}$ is as follows:

Upon receiving the alert from $M G_{c}(h)$, the $M G_{p}(h)$ alerts all upstream unicasting nodes of node ' $S$ ', which belongs to multicast group ' $M G_{p}$ '. Then upstream unicasting nodes of the multicast group ' $M G_{p}$ ', which are upstream nodes to node $S$ balance their outflow load and define $n d(o l)$ then informs the same to $M G_{p}(h)$ .Afterwards the $M G_{p}(h)$ measures $m g d(o l)_{M G_{p}}$ and verifies it as follows:

${ }^{m g d}(o l)_{M G_{p}} \geq d(o l)+\bar{\varepsilon}$

If above equation is true then MGOL process ends at $M G_{p}$, if not that continues to next multicast group in the upstream level of the $M G_{p}$

This process continues till victim node $i$ is free from congestion or if MGOL applied at all upstream multicast groups of the ' $M G_{c}$ '.

The process described above is attempting to avoid the congestion by balancing the outflow load between multicast groups and the same can be referred as Multicast Group level Outflow Load-balancing (MGOL).

Once the MGOL ends then the source multicast group evaluates the $d(o l)$. Based on this ' $d(o l)$ ' value, the transmission source node balances its outflow load. 
Multicast Group Outflow Load-balancing (MGOL) Algorithm $P 1$

$\varepsilon_{M G_{C}}=\frac{\sum_{k=1}^{\left|M G_{C}\right|} m g d(o l)_{M G_{c}}-d(o l)_{k}}{\left|M G_{c}\right|}$

If $n d(o l)_{S}>m g d(o l) M G_{C}$ and

$n d(o l)_{S}-m g d(o l)_{M G_{C}} \geq \varepsilon_{M G_{C}}$ begin

$D_{t}(s)=D_{t}(s)+b t$

Here $D_{t}(s)$ is delay time at the node $s$

bt is buffering time threshold

Value of buffering time threshold bt should be decided such that $d(\mathrm{ol})_{s} \geq m g d(\mathrm{ol})_{M G_{C}}+\varepsilon_{M G_{C}}$

Return.

Endif

P2:

Node $S$ alerts multicast group head $M G_{c}(h)$ about the congestion state of the node $i$.

$M G_{c}(h)$ Alerts all upstream unicasting nodes to node $S$ nodes, which belongs to multicast group $M G_{c}$

Each node of $\left\{n_{u 1}, n_{u 2}, \ldots, n_{u k}\right\}_{M G_{C}}$ updates their ' $n d o l$ ' and alerts about the same to $M G_{C}(h)$

$M G_{c}(h)$ Measures $m g d(o l)_{M G_{C}}$ by the subsequent equation:

$\operatorname{mgd}(o l)_{M G_{C}}=\frac{\mid \sum_{k=1}^{\left|M G_{C}\right|} n d(o l)_{k}}{\left|M G_{C}\right|}$

If $m g d(o l)_{M G_{C}}>d o l$ and $\left(m g d(o l)_{M G_{C}}-d o l\right) \geq \bar{\varepsilon}$ begin

Alert: The victim node $i$ is freed from congestion state

Return.

Endif

P3: $M G_{c}(h)$ Alerts $M G_{p}(h)$

$M G_{p}(h)$ Alerts all unicasting upstream nodes to node $S$, which are belongs to multicast group $M G_{p}$

For each upstream unicasting node $\left\{n \mid n \in M G_{p}\right\}$ begin
If $n d(o l)_{n}>m g d(o l)_{M G_{p}}$ and

$n d(o l)_{n}-m g d(o l)_{M g_{p}} \geq \varepsilon_{M G_{p}}$ begin

$d_{t}(n)=d_{t}(n)+b t$

The Value of buffer threshold bt should be decided such that $n d(o l)_{n} \geq m g d(o l)_{M G_{p}}+\varepsilon_{M G_{p}}$

Endif

Find $n d(o l)_{n}$ and send the same to $M G_{p}(h)$

End-of-for each

Then $M G_{p}(h)$ measures $m g d(o l) M G_{p}$

if $\operatorname{mgd}(o l) M G_{p}-d(o l) \geq \bar{\varepsilon}$ and $\bar{\varepsilon}>0$

Alert: Balancing Outflow load at multicast group $M G_{p}$ removed congestion state at node $i$.

Return;

Endif

For each upstream multicast group in sequence

Begin

Consider $M G_{p}$ as $M G_{c}$

Consider immediate upstream multicast group $M G_{p^{\prime}}$ to multicast group $M G_{p}$ as $M G_{p}$

Go to P1

End-of-foreach

$\left\{\mathrm{MG}_{1}\right.$ | transmissioninitiation node is $\operatorname{src}$ and $\left.\operatorname{src} \in \mathrm{MG}_{1}\right\}$ Measures $d(o l)$ as

$d(o l)=\frac{\sum_{i=1}^{|M G|} m g d(o l)_{M G_{i}}}{|M G|}$

The transmission initiated node ' $s r c$ ' that belongs to multicast group ' $\mathrm{MG}_{1}$ ', balances the outflow load such that congestion state will be avoided.

\section{SIMULATIONS AND RESULTS DISCUSSIONS}

The experiments were conducted using NS-2. A simulation network with hops under mobility and count of 50 to 200 has been build. The simulation parameters described in table 1 . Authentication ensures that the buffer is properly allocated to valid packets. The simulation model aimed to compare OLMRP with ODMRP. The performance check of these two carried out against to the metrics explored below 
Table1: Simulation parameters thatare considered for experiments

\begin{tabular}{|c|c|}
\hline Number of nodes Range & 50 to 200 \\
\hline Dimensions of space & 1000 X 1000 \\
\hline Nominal radio range & $250 \mathrm{~m}$ \\
\hline Cache replacement policy & FIFO \\
\hline Multicast Group Size & $10-60$ \\
\hline$b_{t}$ & 0.08 \\
\hline$\varepsilon$ & 0.4 \\
\hline
\end{tabular}

The scalability of Packet Delivery Ratio with respect to the total number of source nodes that participating in routing and the traffic load emerged from them has been evaluated initially. When the source node count is up to 20 , both ODMRP and OLMRP maintained their packet delivery ratio scalability in similar passion (see fig 1).

During the increase in the source node count more than 20, the ODMRP failed to retain its scalability (see fig 1). This is due to rise in the traffic load caused by increase in source nodes, and the congestion due to the overloaded traffic. The OLMRP advantage over ODMRP in packet delivery ratio is observed. An Average of $32 \%$ is the advantage of OLMRP over ODMRP observed in the packet delivery ratio.

\section{Packet Delivery Ratio}

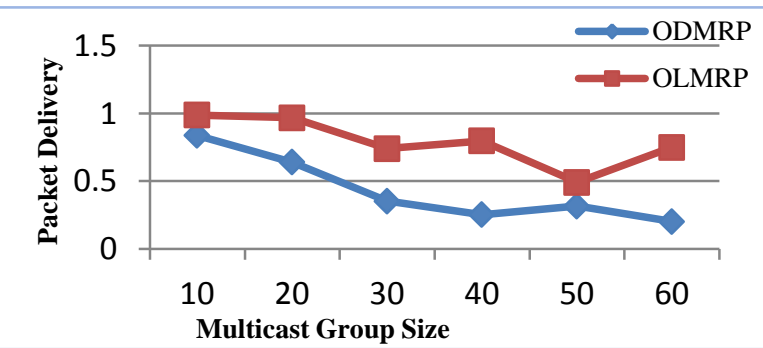

Figure 1: The advantage of OLMRP over ODMRP in Packet Delivery ratio.

Packet Overhead

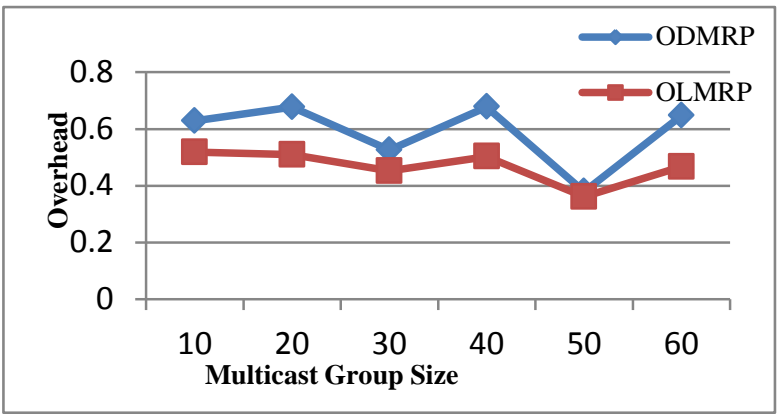

Figure 2: The scalability and stability of OLMRP over ODMRP in packet overhead.
From the evaluation of the Packet Overhead metric (see fig 2) it is evident that the OLMR is stable and scalable than ODMRP. An Averageof 23\% of packet overhead is observed in ODMRP over OLMRP.

\section{CONCLUSION}

This paper discussed a multicast routing algorithm called "Hierarchical Outflow Load-balancing Multicast Routing Protocol for congestion control in Ad hoc Networks" in short referred as OLMRP. This proposed routing strategy aimed to control congestion in hierarchical order, In this regard it first tries to control at hop level outflow load balancing, if failed then attempts to control by group level outflow load balancing, if still not succeed then finally attempts to control the congestion with outflow load balancing between groups. The proposed protocol derived a set of algorithms called Multicast Group Congestion Assessment (MGCA) and Multicast Group Outflow Load-balancing (MGOL). The MGCA algorithm is used to assess the state of the congestion at a group that caused packet dropping. MGOL initiates if the congestion state is observed and controls congestion in hierarchical order. The OLMR is network format independent. Hence it can work with a group of either tree or mesh. As a part of an experimental study, the proposed OLMR compared with ODMRP. The simulation results indicate that the OLMR improved the PDR and minimized the Packet overhead of ODMRP in order of magnitude. With the motivation gained from simulation results of proposed routing topology OLMRP, the future direction can be minimizing the energy usage in OLMRP implementation.

\section{REFERENCES}

[1] Michael Gerharz, Christian de Waal, and Matthias Frank, "A Practical View on Quality-of-Service Support in Wireless Ad Hoc Networks", BMBF

[2] Xiaoqin Chen, Haley M. Jones, A .D .S. Jayalath, "Congestion-Aware Routing Protocol for Mobile Ad Hoc Networks", IEEE, 2007

[3] HongqiangZhai, Xiang Chen, and Yuguang Fang, "Improving Transport Layer Performance in Multihop Ad Hoc Networks by Exploiting MAC Layer Information”, IEEE, 2007.

[4] Yung Yi, and Sanjay Shakkottai, "Hop-by-Hop Congestion Control Over a Wireless Multi-Hop Network", IEEE, 2007

[5] Tom Goff, Nael B. Abu-Ghazaleh, Dhananjay S. Phatak and RidvanKahvecioglu, "Preemptive Routing in Ad Hoc Networks", ACM, 2001

[6] Xuyang Wang and Dmitri Perkins, "Cross-layer Hopbyhop Congestion Control in Mobile Ad Hoc Networks", IEEE, 2008

[7] DzmitryKliazovich, FabrizioGranelli, "Cross-layer Congestion Control in Ad hoc Wireless Networks," Elsevier, 2005

[8] Duc A. Tran and Harish Raghavendra, "Congestion Adaptive Routing in Mobile Ad Hoc Networks", 2006

[9] Nishant Gupta, Samir R. Das. Energy-Aware OnDemand Routing for Mobile Ad Hoc Networks, OPNET Technologies, Inc. 7255 Woodmont Avenue Bethesda, MD 20814 U.S.A., Computer Science Department 
SUNY at Stony Brook Stony Brook, NY 11794-4400 U.S.A.

[10] Laura, Energy Consumption Model for performance analysis of routing protocols in MANET,Journal of mobile networks and application 2000.

[11] LIXin MIAO Jian -song, A new traffic allocation algorithm in AD hoc networks, "The Journal of China University of Post and Telecommunication", Volume 13. Issue3. September 2006.

[12] Chun-Yuan Chiu; Wu, E.H.-K.; Gen-Huey Chen; "A Reliable and Efficient MAC Layer Broadcast Protocol for Mobile Ad Hoc Networks," Vehicular Technology, IEEE Transactions on , vol.56, no.4, pp.2296-2305, July 2007
[13] Giovanidis, A. Stanczak, S., Fraunhofer Inst. for Telecommun., Heinrich Hertz Inst., Berlin, Germany This paper appears in: 7th International Symposium on Modeling and Optimization in Mobile, Ad Hoc, and Wireless Networks, 2009. WiOPT 2009

[14] Outay, F.; Vèque, V.; Bouallègue, R.; Inst. of Fundamental Electron., Univ. Paris-Sud 11, Orsay, France This paper appears in: 2010 IEEE 29th International Performance Computing and Communications Conference (IPCCC)

[15] Yingqun Yu; Giannakis, G.B.; , "Cross-layer congestion and contention control for wireless ad hoc networks," Wireless Communications, IEEE Transactions on , vol.7, no.1, pp.37-42, Jan. 2008. 